\title{
Clitoral HOOD granulosa cell tumor; case report and review of literature
}

\begin{abstract}
Background: Granulosa Cell tumors (GCT) are a sex-cord stroma tumor comprising of $1-2 \%$ of ovarian malignancies. Derived from Schwann cells, GCTs are comprised of granulosa cells, a cell of the ovarian stroma. Patients with these tumors present with signs of increased estrogen such as vaginal bleeding, irregular menstruation and rarely hirsutism and virilization. ${ }^{8,10-12}$ The majority of GCTs are found in the skin, subcutaneous tissue and submucosa with a handful of case reports discussing primary GCTs in the clitoris. ${ }^{3,7}$ Surgery is required for definitive tissue diagnosis and staging. In this case report, we present a case with tissue diagnosis for granulosa cell tumor found on the clitoral hood.
\end{abstract}

Case: A 56-year-old, female, G6P3033 initially presented for evaluation of persistent clitoral cyst for $>2$ years Initial evaluation was significant for $1 \mathrm{~cm}$ clitoral cyst that was firm, smooth and fluctuant with no irregular borders; with worsening vulvodynia. The patient underwent clitoral hood mass excision. The mass was excised entirely and the postoperative recovery was uncomplicated. Pathology findings were significant for completely excised granulosa cell tumor; margins were negative for tumor. Immunohistochemical stain was positive for CD56, CD68, Vimentin, and S-100, and negative for AE1/13, CD31, CD34, SMA, and Desmin.

Conclusion: When evaluating chronic, persistent, or recurrent masses, it is important to take into consideration both benign and malignant causes. Definitive diagnosis is established pathologically. Patients with confirmed GCT should be thorough evaluated pre-operatively with blood work and appropriate imaging. Upon diagnosis, patient with GCT should be regularly followed for recurrence and surveillance.
Volume 12 Issue 4 - 202 I

\section{Neil Patel MD,Alexandra Hamilton MD, Natasha Fievre MD FACOG \\ Department of Obstetrics and Gynecology, The Brooklyn Hospital Center, USA}

Correspondence: Neil Patel, MD, Department of Obstetrics and Gynecology, The Brooklyn Hospital Center, I2I Dekalb Ave. Bk, NY, I I 20I, USA, Tel 303-919-7668, Email N.D.Patel@iCloud.com

Received: July 2I, 202I | Published: July 30, 2021

Keywords: granulosa cell tumor, clitoral hood mass, sex-cord stroma tumor

\section{Abbreviations: GCT, granulosa cell tumor}

\section{Introduction}

Granulosa Cell tumors (GCT) are a sex-cord stroma tumor comprising of $1-2 \%$ of ovarian malignancies. GCTs can occur at any age but have a predilection for postmenopausal women in the fifth decade of life. ${ }^{1-3}$ Derived from Schwann cells, GCTs are comprised of granulosa cells, a cell of the ovarian stroma. These cells are hormonally active and secrete sex steroids such as estrogen. Patients with these tumors present with signs of increased estrogen such as vaginal bleeding, irregular menstruation and rarely hirsutism and virilization..$^{1-4}$ The majority of GCTs are found in the skin, subcutaneous tissue and submucosa with a handful of case reports discussing primary GCTs in the clitoris. ${ }^{5,6}$ Most cases are benign with only $2 \%$ being malignant. ${ }^{5,7}$ There is no known genetic predisposition to GCT. The typical gross appearance of GCT is of tan-yellow appearance owing to steroid production. Microscopically, GCT is made up of granulosa cells - small, pale, round cells with "coffee bean" nuclei and eosinophilic cytoplasmic granules. ${ }^{2,3,6,8}$ Surgery is required for definitive tissue diagnosis and staging. In this case report, we retrospectively studied the clinical presentation and histopathology in a patient with a clitoral hood granulosa tumor. The literature is limited on primary granulosa cell tumors of the clitoral hood, which is evidence to the rarity of the finding.

\section{Case presentation}

\section{Initial presentation}

A 56-year-old, female, G6P3033 initially presented to the clinic for evaluation of persistent clitoral cyst for $>2$ years. Patient stated the cyst had been growing over time, resulting in worsening vulvodynia. Initial evaluation was significant for $1 \mathrm{~cm}$ clitoral cyst that was firm, smooth and fluctuant with no irregular borders. The patient endorsed mild tenderness. Exam showed grossly normal vaginal tissue and was negative for edema, erythema and significant skin discoloration. Uterus and adnexal masses were not palpable. Patient denied weight loss, fatigue, cachexia, fever which are clinical signs and symptoms of malignancy.

Gynecologic history: Uterine fibroids, dysmenorrhea, who underwent laparoscopic hysterectomy (without oophorectomy, secondary to uterine fibroids), no history of abnormal pap smears.

Obstetric history: 3 Full term deliveries via cesarean section. 3 early trimester losses.

Medical history: Hypertension, Hypercholesterolemia, Diabetes.

Mellitus surgical history: Cesarean Section (x3), Dilation and Curettage (x3), Laparoscopic Hysterectomy. 
Familial history: patient denies significant family history, abnormal familial gynecologic disorders, diseases, cancers.

Medications: Metoprolol, Simvastatin, Metformin, Vaseretic.

\section{Operative findings}

Patient underwent elective surgical management for clitoral hood mass excision with no significant intra-operative findings. Evaluation was consistent with initial presentation. The mass was excised entirely, and the post-operative recovery was uncomplicated.

\section{Pathology findings}

Pathology findings were significant for completely excised granulosa cell tumor; margins were negative for tumor. Immunohistochemical stain was positive for CD56, CD68, Vimentin, and S-100, and negative for AE1/13, CD31, CD34, SMA, and Desmin.

\section{Discussion}

Granulosa cell tumors are mostly asymptomatic, benign, slow growing tumors with preponderance in adult females. ${ }^{9}$ Most benign GCTs measure $<3 \mathrm{~cm}$ in size, and commonly found in women aged 40-60years. ${ }^{3}$ Granulosa cell tumors can be found in skin, subcutaneous tissue and submucosa and the majority of lesions present as a single mass. Ovarian GCTs often present with signs of excess estrogen and when large, may cause obstructive symptoms. ${ }^{3}$ Vulvar GCTs often present as a slowly enlarging subcutaneous, mobile mass that may present with pruritis and hyperpigmentation. ${ }^{10}$ Microscopically, GCTs are distinguished by the presence of granulosa cells - small, pale, round cells with coarse, eosinophilic cytoplasmic granules which stain positive for neural markers such as $\mathrm{S} 100 .{ }^{3,7,9}$ To date, few cases of clitoral granulosa cell tumors have been reported.

Our patient presented with a clitoral mass that persisted for $>2$ years. On observation the mass initially was $1 \mathrm{~cm} \mathrm{x} 1 \mathrm{~cm}$, firm with mild tenderness. Prior to the excision, patient underwent transvaginal ultrasound evaluation one month prior to procedure; status posthysterectomy, both ovaries identified, no cysts or adnexal masses noted. The mass excised was sent to pathology and was significant for granulosa cell tumor with clear margins. Immunohistochemistry stained positive for S-100 protein, CD68 and vimentin, which further strengthened the schwannian origin. ${ }^{5,8,9}$ This condition requires differentiation from other benign disease of the external genitalia.

The gold standard of treatment is complete resection with routine follow up for recurrence. ${ }^{3,5}$ The recurrence rate with negative resection margins for benign GCTs is $2 \%-8 \%$. In addition, extragenital areas should also be evaluated as studies support cases of multiple GCTs at various sites. ${ }^{3,5,11}$ There is ongoing debate regarding treatment for GCTs as most studies indicate that radiation and chemotherapy have not been successful. ${ }^{10,12}$ A study by Golchai J et al. however, found that neoadjuvant chemotherapy successfully treated GCT of the uterine cervix. ${ }^{11}$ This discrepancy illustrates the need for more research and development of treatment guidelines for GCTs.

\section{Conclusion}

When evaluating chronic, persistent, or recurrent masses, it is important to take into consideration both benign and malignant causes. Definitive diagnosis is established pathologically. Patients with confirmed GCT should be thorough evaluated pre-operatively with blood work and appropriate imaging. Upon diagnosis, patient with GCT should be regularly followed for recurrence and surveillance.

\section{Acknowledgments}

None.

\section{Funding}

None.

\section{Conflicts of interest}

The authors did not report any potential conflicts of interest.

\section{References}

1. Pankratz E, Boyes DA, White GW, et al. Granulosa cell tumors: a clinical review of 61 cases. Obstet Gynecol. 1978;52(6):718-723.

2. Schumer ST, Cannistra SA. Granulosa cell tumor of the ovary. Journal of clinical oncology. 2003;21(6):1180-1189.

3. Shim SH, Lee SJ, Kim DY, et al. A long-term follow-up study of 91 cases with ovarian granulosa cell tumors. Anticancer research. 2014;34(2):10011010

4. Pectasides D, Pectasides E, Psyrri A. Granulosa cell tumor of the ovary. Cancer Treat Rev. 2008;34(1):1-12.

5. Goel G, Singh N, Gupta R, et al. Recurrent benign cutaneous granular cell tumor: A case report. J Cytol. 2013;30(4):287-288.

6. Min JW, Kim YS. A case of granular cell tumor of the clitoris in a postmenopausal woman. J Menopausal Med. 2017;23(2):135-137.

7. Elkousy H, Harrelson J, Dodd L, et al. Granular cell tumors of the extremities. Clin Orthop Relat Res. 2000;380:191-198.

8. Goel A, Narian A, Singh K, et al. Granular cell tumor of clitoris: a case report. Internal Journal of Reproduction, Contraception, Obstetrics and Gynecology. 2019;8(5):2146-2148.

9. LeBH, Boyer PJ, Lewis JE, et al. Granularcell tumor: immunohistochemical assessment of inhibin-alpha, protein gene product 9.5, S100 protein, CD68, and Ki-67 proliferative index with clinical correlation. Arch Pathol Lab Med. 2004;128(7):771-775.

10. Papalas J, Shaco-Levy R, Robboy S, et al. Isolated and synchronous vulvar granular cell tumors: a clinicopathologic study of 17 cases in 13 patients. Int J Gynecol Pathol. 2010:173-180.

11. Golchai J, Zargari O, Paknejadi MB. Multiple granular cell tumor in a teenager: Report of a case and review of the literature. Acta Med Iran. 2004;42:228-231

12. Koukourakis GV, Kouloulias VE, Koukourakis MJ, et al. Granulosa cell tumor of the ovary: tumor review. Integr Cancer Ther. 2008;7(3):204215 . 